\title{
Targeted Alpha Therapy Trial in Bangladesh: Promise for Advanced MUC1 - Expressing Tumors \\ $\mathbf{1}_{\text {Lutfun Nisa, }}{ }^{2}$ Kamila Afroj Quadir, ${ }^{1}$ Shamim MF Begum, ${ }^{\mathbf{1}}$ Raihan Hussain and ${ }_{\text {Mizanul Hasan }}$ \\ ${ }^{1}$ National Institute of Nuclear Medicine \& Allied Science (NINMAS), Dhaka \\ ${ }^{2}$ Bio-Science Division, Bangladesh Atomic Energy Commission, Dhaka
}

Correspondence Address: Former Prof. Lutfun Nisa, National Institute of Nuclear Medicine \& Allied Science, Block -D BSMMU Campus, Shabagh, Dhaka, Email: nisa.lutfun@gmail.com

\section{ABSTRACT}

Introduction: Targeted alpha therapy (TAT) is a new experimental therapy that targets cancer cells and tumor capillary endothelial cells through intravenous injection of an alpha immuneconjugate (AIC). The AIC is formed by labeling the cancer targeting vector (monoclonal antibody) with alpha emitting radioisotopes using a bifunctionalchelator. The monoclonal antibody (MAb) is raised against antigens (e.g. MUC1) over-expressed on the surface of certain cancer cells. There are several centers notably in Europe, the US and Australia that are actively involved in TAT clinical trials of different cancers using a variety of techniques, alpha emitters and MAbs. Observations from their cumulative experience suggest that TAT is safe and effectivebut needs further trials for practical acceptance. Especially critical is the issue of maximum tolerance dose (MTD) which needs to be established for maximum target kill. Bangladesh has the infrastructure to conduct aTAT clinical trial and can significantly add to the growing pool of data for advanced treatment of cancers through collaborative involvement in targeted alpha therapy research.

Objective: The aim of the article is to present a general overview of targeted alpha therapy and to discuss the feasibility of a TAT clinical trial in Bangladesh in the context of current cancer management situation in the country.

Method: Literature review of significant publications was done to obtain an update of the current status of targeted alpha therapy. Relevant issues of TAT are presented for a theoretical basis of the technology. Next, the methodology of a proposed clinical trial is discussed, together with the practicability of its introduction in Bangladesh.

Conclusion: Implementation of TAT clinical trial will help to develop an advanced technology and build- up skilled manpowerin Bangladesh.It will optimize the key parameters of targeted alpha therapy, $i$ e stability and specific activity of the alpha-conjugate and establish the maximum tolerance dose for the AIC. If the clinical trial is successful, it can change the prognosis of many end-stage cancers. Patients in Bangladesh
\end{abstract}

with advanced MUCI expressing tumors of the breast, ovary, pancreas and prostate can have some measure of hope with stability of the disease.

Key Words: Targeted alpha therapy, monoclonal antibody, radioimmunoconjugate, $213 \mathrm{Bi}-\mathrm{C595}$

\section{INTRODUCTION}

Cancer is a major challenge all over the world and particularly in Bangladesh where the load is reported to be more than 1,200,000. It has been identified as the 6th cause of mortality in Bangladesh with more than half of the cancer patients dying within five years of diagnosis. (National Cancer Control Strategy Plan of Action 20092015). As in most developing countries, cancer therapy in Bangladesh includes surgery, radiation, chemotherapy, hormonal therapy, immunotherapy, radioiodine therapy or a combination of two or more of these. In metastatic disease chemotherapy and external beam radiation therapy may show clinical improvement but long term survival is uncertain due to the presence of micrometastasis. The cytotoxic drugs used to target rapidly dividing cancer cells also destroys certain normal tissues, resulting in toxicities like alopecia, gastrointestinal symptoms, myelosuppression etc. Hence the search for new, selective anticancer agents that differentiates between malignant and nonmalignant cells. New development and insights into cancer biology has paved the way for personalized cancer management that may be influenced by patient ethnicity, sex and tumor histology (1). Thus a particular therapy may be effective in patients whose cancers have a specific 
molecular target, but ineffective in the absence of such a target (2). This specificity towards a particular cancer is the essence of targeted therapy. There are different types of targeted therapies. Some target the inner parts of the cells, sometarget the receptors on the cell surface (through specific monoclonal antibodies) and others like the antiangiogenesis therapy, targets the blood vessels that supply nutrients to the cancer cells. Targeted therapies, particularly those that include monoclonal antibodies have significantly changed the approach towards cancer management in the past decades. Cristine Craig (3) has aptly stated:'Cancer researchers and clinicians familiar with targeted nuclear medicine have envisioned a time when the power within the nucleus could be harnessed for targeted radio-immunotherapies against cancer cells within the human body and especially against occult cancers, micrometastases, and minimal residual disease remaining after completion of surgery, chemotherapy, and other treatments'.

This article presents a general overview on targeted therapy and discusses the practicality of a TAT clinical trial in Bangladesh in the context of current cancer management situation in the country.

\section{Monoclonal Antibodies (MAbs)}

Monoclonal antibodiesare mono-specific antibodies are produced by clones of identical immune cells. The technique by which monoclonal antibodies are produced is called the hybridoma technology which was discovered in 1975 by Georges Kohler of West Germany and Cesar Milstein of Argentina. For their work, both scientist were awarded the 1984 Noble prize jointly with Niels Jerne of Denmark for physiology and medicine (Wikipedia).

The monoclonal anitibodies raised against cancer antigens were found to mediate antibody dependent cell-mediated cytotoxicity (4) and this discovery opened the way for major development in cancer therapeutics. Since the time it became feasible to produce MAbs for therapeutic use more than three decades ago (5) a new clinical field of radio-immunotherapy (RIT) was developed. In the process, the monoclonal antibody

industry expanded exponentially with current valuation at billions of dollars. Innumerable number of MAbs continue to be raised against antigens that are over expressed by several types of cancer cells. Approximately 30 of these monoclonal antibodies have been approved by FDA for use in clinical practice and many more are currently being tested in clinical trials (6).

\section{Radionuclides}

Radionuclides are the major players in radioimmunotherapy. However the suitabilityof a radionuclide for therapy depends on a number of factors, namely its physical and chemical properties, its fate after antibody metabolism in vivo, and the nature of the radiation such as low or high linear energy transfer (LET) emission (7). Three types of particulate radiation are of consequence for targeted radionuclide therapy: $\beta$-particles, $\alpha$-particles and Auger electrons, which can irradiate tissue volumes with multicellular, cellular and subcellular dimensions, respectively. Moreover, there are multiple radionuclides decaying by each of these three modes with a variety of tissue ranges, half-lives and chemistries, offering the attractive possibility of tailor-making the properties of a targeted radionuclide therapeutic agent to a particular type of cancer and perhaps, ultimately, the needs of an individual patient

(8). The monoclonal antibodies in RIT are conjugated to radionuclides which provide a specific internal radiotherapy. Clinical success has been achieved with the $\boldsymbol{\alpha}$-emitting (electrons) nuclides ${ }^{90} \mathrm{Y}$ and ${ }^{131} \mathrm{I}$, conjugated to anti-CD20 MAb in follicular B-cell nonHodgkin lymphoma (9). Despite the predominant use of a-emitters in radio-immunotherapy trials, investigators have long recognized the potential advantages of $\alpha$-particle emitters (10). Alpha-particles are positively charged heliumnuclei with a shorter range $(50-80 \mathrm{~m})$ and higher energy $(5,000-8,000 \mathrm{keV})$ than $\beta$-particles. Alpha-particles differ from $\beta$-particles in energy $(\mathrm{MeV})$, tissue range, linear-energy transfer (LET) and number of DNA hits needed to kill a cell. A large number of in-vitro and in- vivo experiments with 
a-immunotherapy have shown dramatic superiority over $\alpha$-immunotherapy, as only a few alpha hits of the nucleus are needed to achieve $63 \%$ cell kill $(11,12,13)$.

The unique physical and radiobiologic properties of $\alpha$ particles, such as short path length and high LET offers the potential for more specific tumor cell killing with less damage to surrounding normal tissues $(14,10)$. Thus $\alpha$ radiation is ideal for killing isolated cancer cells in transit in the vascular and lymphatic systems, as well as in regressing tumors by disruption of tumor capillary networks by targeting and killing tumor capillary endothelial cells. The LET is typically $100 \mathrm{keV} / \mu \mathrm{m}$, giving a higher probability of causing double strand breaks. The ratio of single strand break/double strand break 220 , is compared to 60 for low LET radiation $(15,16)$. Of the many processes involved in destruction of cancer cells, apoptosis and mitotic catastrophe are the two major routes for cell deaths induced by radiation $(17,18)$. It also initiates over expression of p53 leading to delays in the G1 phase of the cell cycle (19).A programmed sequence of events leads to the elimination of cells without releasing cytotoxic substances into the surrounding tissues. Thus apoptosis plays a crucial role in developing and maintaining healthy tissues by eliminating old cells, unnecessary cells, and unhealthy cells (15). Currently the $\alpha$-radionuclides of interest are $\mathrm{Bi}-212, \mathrm{Bi}$ 213, At-211 and Ac-225.

\section{Targeted $\alpha$-immunoconjugate}

The immunoconjugate is a complex that consist of three separate components: an antibody that binds to a cancer cell antigen with high specificity, an effector molecule that has a high capacity to kill the cancer cell, and a linker that will ensure the effector does not separate from the antibody during transit and will reliably release the effector to the cancer cell or tumourstroma. (20). The high affinity antibody-antigen interaction allows specific and selective delivery of a range of effectors, including pharmacologic agents, radioisotopes, and toxins, to cancer cells (20). The alpha immunoconjugate (AIC) is similarly formed by labeling the cancer targeting monoclonal antibody (MAb) with the $\alpha$-emitting radioisotope using a bifunctionalchelator (15). The monoclonal antibody becomes the targeting vector that takes the alpha particles to the targeted cancer cells (15).

\section{Clinical Applications of TAT}

Monoclonal antibodies or proteins labeled with alpha emitters such as Bi-212, Bi-213, At-211 and Ac-225 has been extensively studied for more than two decades. Subsequently, the cytotoxic effect of TAT on cancer cells demonstrated in both in-vitro and in- vivo experimental models progressed on to clinical trials (21). The AIC may be administered either by intravenous, intracavitory and/or by intralesional route depending on the specific clinical requirement. After intravenous injection, the AIC first targets isolated cancer cells circulating in the lymphatic and vascular system, second, it acts on cell clusters and regresses metastatic cancers. Last but not the least, it targets the tumor capillary endothelial cells and disrupts the vasculature of solid tumors Thus minimal residual or micrometastatic disease can be potentially eliminated as well as solid tumors $(15,22,23)$. A phase I study of toxicity and response of systemic alpha therapy for metastatic melanoma using the alpha-immunoconjugate (213)Bi-cDTPA-9.2.27 MAb )by Raja et. al. reports positive results. Most patients in their study showed reductions of the tumor marker melanoma inhibitory activity protein (MIA) over eight weeks and toxicity at any level was not observed over the range of administered activities. The authors concluded that observation of responses without any toxicity indicated that TAT has the potential to be a safe and effective therapeutic approach for metastatic melanoma (24). Other phase 1 trials for acute myeloid leukemia (AML) was completed at Memorial Sloan Kettering Cancer Center (25) and for bone metastasis in Norway (26). Additional clinical trials included phase I/II trial for post-chemotherapy of AML, systemic trial for metastatic melanoma, glioblastoma and for lymphoma $(24,27,28)$. These investigators also reported positive results without any untoward side effects or toxicities. 
Compared to the intravenous administration of AIC, the intralesional and intracavitory route delivers higher dose to the tumor and minimizes risk to critical organs like the bone marrow. It also reduces the risk of unknown toxicity due to unforeseen microscopic accumulation of the AIC elsewhere in the body (9). In a phase I trial of stage 4 melanoma patients, Allen et al. found intralesional TAT to be quite safe up to $450 \mathrm{mCi}$, and efficacious at a dose of $200 \mathrm{mCi}$. The authors observed significant decline in serum marker melanoma-inhibitory-activity protein at 2 weeks postTAT. Together with the apoptosis and ki67 proliferation marker tests intralesional TAT appeared to be a promising therapy for the control of inoperable secondary melanoma or primary ocular melanoma (29). Administration of 211At labeled monoclonal antibody [211 At-MX35 F( $\left(\mathrm{ab}^{\prime}\right) 2$ ] through intraperitoneal route in a phase 1 study of metastatic ovarian cancer showed that it is possible to achieve therapeutic absorbed doses inmicroscopic tumor clusters without significant toxicity (30). Similarly, the regional administration of 211At labeled anti-tenascin monoclonal anti-body 81C6 (ch81C6) into surgically created resection cavity in recurrent malignant brain tumors was found to be feasible, safe and associated with a promising antitumor benefit in patients with malignant central nervous system tumors (31).

Tumor regression obtained from systemic TAT was attributed to the killing of tumor capillary endothelial cells. The targeting of tumor capillaries in solid tumors subsequently came to be known as the tumor anti-vascular alpha therapy (TAVAT) and this concept holds promise in the treatment of many solid tumors that over expression antigens against which specific MAbs are available (15).

\section{C595 anti-MUC1}

Mucin1 (MUC1) is a highly O-glycosylated, transmembranemucin-type glycoprotein (32). It is expressed in small amounts on the apical membrane of various types of normal epithelial cells and on the surface of different cell types of hematopoietic origin
(33). During malignant transformation, the expression and distribution of MUC1 undergoes drastic change with over expression and depolarized distribution in several epithelial malignancies (34). Cancer-associated MUC1 is structurallydifferent to normal MUC1 in that the former has shorter and less dense O-glycan chains, which exposes novelregions of the protein core.In particular MUC1 is found to be frequently up-regulated and abnormally glycosylated in a number of common malignancies, including breast, bladder, colon, ovarian, prostate and gastric cancer. It was found that over $90 \%$ of primary prostate, pancreatic and ovarian tumours expressed MUC1 while 95\% of normal tissues did not $(35,36,37)$. Further, MUC1 expression was found on the surface of cancer cell lines. High expression of the MUC1 is closely associated with cancer progression and metastasis leading to poor progression. Thus mucins have an important role in pathological state with immense potential not only as diagnostic or prognostic markers but also as therapeutic targets (38). These findings points towards the exciting possibility of targeted alpha immunotherapy of MUC1 expressing tumors.

The $\mathrm{C} 595$ is an $\mathrm{IgG3}$, murine MAb raised against the protein core of human urinary epithelial MUC1 (36). Many cancers and in particular pancreatic and prostate cancer sections show strong staining using the C595 monoclonal antibody against MUC1. The expression of tumour-associated antigen MUC-1 on breast, prostate, ovarian and pancreaticcancer cell lines, in cell clusters and animal xenografts was detected by indirect immmunostaining (39).

The C595 MAb, raised against MUC1 by Price et. al. (40) was chelated with the bifunctional binding molecule CHX-Aand labeled with Bi-213 alpha emitter to form the alpha-immunoconjugate (41). The role of this AIC for control of cancers in-vivo has been investigated for stability, labeling yield, toxicity, cytotoxicity and response in preclinical TAT studies for breast, ovarian, prostate and pancreatic cancers. These preclinical results showed inhibition of tumour growth 
and regression of cell clusters. The lethal pathway in all in- vitro studiesafter TAT was found to be predominantly by apoptosis (42).

In pancreatic cancers, systemic TAT has the potential to regress and to eliminate micro--metastases. It is expected that TAT would be indicated for the control of pancreatic cancer after resection of the primary tumor, when complete cell kill of micrometastases could be achieved, leading to elimination of clinical disease. The radioimmunoconjugate 213Bi-C595 is the agent most suitable for pancreatic cancers that are positive for over expression of MUC1 antigen (39). Multiple clinical trials of targeted radionuclide therapy of pancreatic cancer performed in the last decade demonstrated safety and potential efficacy of radionuclide therapy for treatment of this formidable disease (43).

\section{Dosing and Effectiveness}

Determination of the maximum tolerance dose (MTD) is a very critical issue for cancer regression. Therefore proper dosing and the effectiveness of the dose selected in targeted alpha therapy are of particular concern. Clinical trials undertaken for acute AML has determined

the MTD with $213 \mathrm{Bi}-\mathrm{AIC}$ at $1 \mathrm{mCi} / \mathrm{kg}$. For intra-cavity therapy of GBM with 211 AT-IC was determined at $\sim 0.14 \mathrm{mCi} / \mathrm{kg}$. For metastatic melanomas, the MTD has not been established, though the efficacious dose for some melanomas was found to be less than $0.3 \mathrm{mCi} / \mathrm{kg}(22)$.

The tumor regression in the phase I clinical trial of systemic TAT of metastatic melanoma is attributed to TAVAT which could change the prognosis for many endstage cancers $(44,45)$. However the efficacy of TAVAT needs to be studied further with determination of the MTD. Establishing the MTD for targeted therapy of a particular cancer type is imperative for practical acceptance. Fractionization of the total calculated dose may give improved efficacy as tumor capillaries may be damaged, resulting in increased extravascular permeability for the AIC. Since toxicity and efficacy are the relevant end points for treatment evaluation, the definition of all toxicities at different dose levels is crucial. Though targeted therapy often do not cause significant haematologic toxicity, with higher doses the scenario may be different and there is always the possibility of nephrotoxicity. Dose limiting toxicity of TAT is therefore defined in terms of renal function: GFR and serum creatinine.

\section{Microdosimetry}

Microdosimetry is the measurement of the effects of very small amounts of ionizing radiation, especially along the single track of such radiation. It is important not only for radiation protection but also for understanding the mechanisms of radiation action and for assessing the radiation risk (46). TAT has the advantage of giving therapeutic doses to individual cancer cells and low dose radiation to distant normal cells. However, because of the very short range of alpha radiation, tumor or organ dose is difficult to estimate. Microdosimetry technique can measure the microscopic distribution of energy from different types of radiation. Using the Monte Carlo models for biological dosimetry, the location and form of radionuclides within cells can be used to estimate radiation dose distributions and effects (47).

\section{Feasibility and Rationale for TAT in Bangladesh}

The aim of a TAT clinical trial in Bangladesh is to assess toxicity and response of systemic alpha therapy for metastatic breast, ovary, prostate and pancreatic cancers with high expression of MUC1. Many centers around the world, notably in Australia, the USA and Europe are using targeted alpha therapy (TAT) in selected cases. Clinical trials at these centers have demonstrated the safety, feasibility and activity of targeted alpha particle therapy in the treatment of small volume and cyto-reduced disease. Bangladesh can significantly add to this growing pool of data for advanced treatment of cancers through such collaborative involvement. In the process a new technology like targeted alpha radio-immunotherapy will be established, together with improved development of microdosimetry and 
immunohistiochemistry techniques. More importantly, it will contribute to the modernization of laboratory facilities and the training of manpower in Bangladesh. The main purpose of the study would be to determine the MTD for each patient. The study group will include patients with stage four MUC1 positive cancers having progressive disease. Only those who have either completed or have declined other systemic therapies will be included in the trial. Immunohistiochemistry of tumour samples to document the over-expression of the MUC1 glycoprotein and to assess the immunoreactivity of the cancer cell cluster to C595 for each cell line will determine the final selection of patients.

The Actinium: Bismuth generator system (produced by the Institute for Transuranium Elements (ITU), Karlsruhe, Germany) will be the source for the $\alpha$ - particle emitting radionuclide $\mathrm{Bi}-213$. The monoclonal antibody $\mathrm{C} 595$ against MUC1 and the Ac-225:Bi-213 generator for the study will be obtained through collaboration with Australia. The method of preparing the alpha radionuclide by chelating 213Bi to the monoclonal antibody (MAb) C595 and the modus perandi for the experiment will be acquired through technical expertise from Australia. Cohorts of 3 subjects with end-stage cancer will be treated with escalating doses of $5 \mathrm{mCi}$, then $10,15,20,25,30$ $\mathrm{mCi}$ every 2 months. If adverse events are seen in one patient then the maximum tolerance dose will be the preceding dose.Patients will be followed up for 12 months with emphasis on the detection of delayed radiation nephrosis. The response to therapy will be assessed by physical examination of the patient, imaging of tumors, pathology comparisons over 12 weeks, glomerular filtration rate, computed tomography comparisons, and changes in tumor markers over 8 weeks. Responses will be based on the Response Evaluation Criteria in Solid Tumors [RECIST] (48).

\section{Outcome}

The proposed study will bring together the highly selective features of a unique targeting system with the high cytotoxicity of alpha particles for treatment of MUCI expressing tumors of the breast, ovary, pancreas and prostate.It will optimize the key parameters of targeted alpha therapy, ie stability and specific activity of the $\mathbf{\alpha}$-conjugate and maximum tolerance dose that may lead to a much higher rate of tumor control. The implementation of TAT technology will therefore be a significant step towards modernization of cancer therapy in Bangladesh. In addition, it will pave the way for further advances in targeted therapy with new radioisotopes, antibodies and procedures. More importantly, TAT will offer a treatment option in a particular subset of cancer patients with advanced disease in whom current treatment options have proved ineffective. Targeted alpha therapy therefore holds promise in cancer therapeutics especially in Bangladesh where the five year cancer survival rate is low. This technique can give hope to many end-stage cancers patients with no other option for treatment. In advanced MUC1 receptor positive cancers of the breast, ovary, pancreas and prostate, TAT can deliver high cytotoxicity to targeted cancer cells that has metastasized and disseminated throughout the body. The benefits of such agents would include a higher therapeutic index and lower toxicity than conventional therapies and thereby contribute to significantly improved cancer health care in Bangladesh.

\section{Limitations}

The main limitation of the TAT trial in Bangladesh will be the complexity and high technological nature of the study as well as the sine qua non for multidisciplinary team work. With regard to dosimetry, it is particularly complicated because of inhomogeneous distribution of the $\alpha$-particles in tumor and tissues. Therefore the dosimetry technique for $\alpha$-particles needs to be developed in Bangladesh through state- of- the- art biological dosimetrymodels.

Patients will require repeat laboratory testing and biopsies of the tumours which may be inconvenient. Explaining the circumstances of the tests with compassion, care and understanding towards the patient may make these unpleasant situations somewhat acceptable to the patient. 
The effectiveness of the therapy on patients are influenced by a number of factors such as the specificity of the antibody/targeting construct; the level of antigenic expression on the tumor cells; the potential loss of immunoreactivity of the antibody/targeting construct; the amount of unlabeled antibody/targeting construct after injection; the existence of diffusion barriers that hinder the penetration of the antibody/targeting construct into the tumors and too low specific radioactivity. However, all of these can be overcome through technical expertise from the collaborating partner and through meticulous attention to details, as well as dedication and commitment of the local researchers.

\section{CONCLUSION}

Currently there are no systemic treatment that can inhibit the progression of cancer that leads invariably to the death of the patient. TAT has the potential for durable therapeutic responses in stage four cancer patients. It will contribute to significant advancement in cancer therapeutic. According to the Technical Meeting on Alpha Emitting Radionuclides and Radiopharmaceuticals for Therapy conducted by the International Atomic Energy Agency (IAEA) in Vienna in 2013: "TAT will play an important role in the treatment of disseminated, chemoresistant and radioresistant metastatic disease, against which there are no efficacious treatment options. Combined with other therapies, including chemotherapy, signaling pathway inhibitors and even targeted $\beta$ - emitting radionuclides, TAT is likely to improve survival"(15).

\section{REFERENCES}

1. Calvo E, Baselga J. Ethnic differences in response to epidermal growth factor receptor tyrosine kinase inhibitors. J Clin Oncol 2006;24(14):2158-2163.

2. Gerber DE. Targeted therapies: a new generation of cancer treatments. Am Fam Physician 2008;77:311-9.

3. Craig C. Radioisotopes: The Medical Lifesavers That Congress Is Suppressing. 21st Century Science \& Technology Winter 2009/2010.https://www.21stcenturysciencetech.com/.../Isotope Suppression

4. Köhler G, Milstein C. Continuous cultures of fused cells secreting antibody of predefined specificity. Nature. 1975;256(5517):495-497 (cross ref).
5. Milstein Ceasar. With the benefit of hind sight. Trends in Immunology. 2000; 21: 359-364 (cross ref).

6. Justin K.H. Liu.The history of monoclonal antibody development - Progress, remaining challenges and future innovations. Annals of Medicine and Surgery 2014;3 (4): $113-116$

7. Goldenberg DM. Targeted therapy of cancer with radiolabeled antibodies. J Nucl Med 2002;43(5):693-713 (cross ref).

8. Zalutsky MR, Reardonb DA, Pozzia OR, Vaidyanathana G, Bigner DD. Targeted a-particle radiotherapy with 211Atlabeled monoclonal antibodies. Nuclear Medicine and Biology 2007; 34: 779 - 785.

9. Elgqvist J, Frost S, Pouget J-P and Albertson P (2014) The potential and hurdles of targeted alpha therapy - clinical trials and beyond. Front. Oncol.2014; 3:324. doi: 10.3389/fonc.2013.00324.

10. Mulford DA, Scheinberg DA, Jurcic JG. The Promise of Targeted Alpha Particle Therapy. J Nucl Med 2005; 46 (Suppl 1): 199S-204S.

11. McDevitt MR, Barendswaard E, Ma D, Lai L, Curcio MJ, Sgouros G, et al. An alpha-particle emitting antibody. Cancer Res 2000; 60:6095-6100.(cross ref).

12. Allen BJ. Can alpha-immunotherapy succeed where other systemic modalities have failed? Nuclear Med Commun 1999; 20:205-207 (cross ref).

13. Kennel SJ, Boll R, Stabin M, Schuller HM, Mirzadeh S. Radioimmunotherapy of micrometastases in lung with vascular targeted 213Bi. Br J Cancer 1999; 80:175-84.(cross refer).

14. Sgouros G, Roeske JC, McDevittMR, PalmS, Allen BJ, Fisher DR, et al MIRD Pamphlet No. 22 (Abridged): Radiobiology and Dosimetry of $\alpha$-Particle Emitters for Targeted Radionuclide. J Nucl Med 2010; 51(2): 2 311-328.

15. Allen BJ, Huang CY, Clarke RA. Targeted alpha anticancer therapies: update and future prospects. Biologics: Targets \& Therapy 2014;8:255.www.ncbi.nlm.nih.gov/

16. Nunez MI, Villalobos M, Olea N, et al. Radiation-induced DNA double-strand break rejoining in human tumour cells. $\mathrm{Br} \mathrm{J}$ Cancer.1995;71:311-316.

17. Eriksson D, StigbrandT. Radiation-induced cell death mechanisms. Tumour Biol 2010; 31(4):363-372. doi: 10.1007/s13277-010-0042-8. Epub 2010 May 20.

18. Kolesnick RN, Haimovitz-Friedman A, Fuks Z. The sphingomyelin signal transduction pathway mediates apoptosis for tumor necrosis factor, Fas, and ionizing radiation. Biochem Cell Biol 1994;72:471-474(cross ref).

19. Pandita TK, Lieberman HB, Lim DS, et al. Ionizing radiation activates the ATM kinase throughout the cell cycle. Oncogene 2000;19:1386-1391( cross ref).

20. Smaglo B G., Aldeghaither D, Weiner L M. The development of immunoconjugates for targeted cancer therapy. Nature Reviews Clinical Oncology 2014; 11:637-648. 
21. Elgqvist J., (Editor), Targeted alpha therapy. Current Radiopharmaceuticals 4 (2011) (Issues 3 and 4).

22. Allen BJ. Future Prospects of Targeted Alpha Therapy. Current Radiopharmaceuticals 2011;4:336-342.

23. Sofou S, Thomas JL, Lin HY, McDevitt MR, Scheinberg DA, Sgouros G.Engineered liposomes for potential alpha-particle therapy of metastatic cancer. J Nucl Med 2004; 45(2):253-260.

24. Raja C, Graham P, Abbas Rizvi SM, Song E, Goldsmith H, Thompson J, et.al. Interim analysis of toxicity and response in Phase 1 trial of systemic targeted alpha therapyfor metastatic melanoma. Canc Biol Therapy 2007;6:846-852.

25. Jurcic GJ et al. Targeted alpha particle immunotherapy for myeloid leukaemia. Blood 2002; 100(4):1233-1239.

26. Nilsson S, Larsen RH,Fossa SD, Balteskard L, Borch KW, Westlin JE, Salberg G, Bruland OS. First Clinical experience with alpha emitting radium-223 in the treatment of skeletal metastases. Clin Cancer Res 2005;11:4451-4459.

27. Schmidt D. Phase 1 clinical study on alpha-therapy for nonHogkin lymphoma 4th Alpha-Immunotherapy Symp. ed A Morgenstern (Dusseldorf:ITU) 2004;pp 12 (cross ref).

28. Zalutsky MR Current status of therapy of solid tumours: brain tumour therapy. J Nucl Med 2005;46:1515-1565.

29. Allen BJ, Raja C, Rizvi S, Li Y, Tsui W, Graham P, Thompson $\mathrm{JF}$, et.al. Intralesional targeted alpha therapy for metastatic melanoma. Cancer Biol Ther 2005;4(12):1318-1324.

30. Andersson H, Cederkrantz E,Back T, Divgi C,Elgqvist J, Himmelman $\mathrm{J}$ etal. Intraperitoneal alpha-particle radioimmuno therapy of ovarian cancer patients: pharmacokinetics and dosimetry of (211) At-MX35F(ab')2-aphaseIstudy. J Nucl Med 2009;50(7):1153-1160.doi:10.2967/jnumed.109.062604.

31. Zalutsky MR, Reardon DA, Akabani G, Coleman RE, Friedman AH, Friedman HS, etal .Clinical experience with alpha-particle emitting 211 At: treatment of recurrent brain tumor patients with 211At-labeled chimeric antitenas cinmono-clonal antibody81C6. J Nucl Med 2008;49(1):3038.doi: 10.2967/jnumed.107. 046938.

32. GendlerSJ.MUC1, The Renaissance molecule. J Mammary Gland Biol Neoplasia 2001;6:339-353. doi:10.1023/A: 1011379725811.

33. Zotter S, Hageman PC, Lossnitzer A, Mooi WJ, Hilgers J Tissue and tumor distribution of human polymorphic epithelial mucin. Cancer Rev 1988;11/12:55-101.

34. Hollingsworth MA, Swanson BJ. Mucins in cancer: Protection and control of the cell surface. Nature Rev Cancer 2004;4:45-60. doi:10.1038/nrc1251.

35. Song YJ, Qu CF, Rizvi SMA, Li Y, Robertson C, Raja C et al. Cytotoxicity of PA12,C595 and Herceptin vectors labeled with the alpha-emitting radioisotope Bismuth-213 for ovarian cancer cell monolayers and clusters. Cancer Letters 2006;234:2176-2183.
36. Allen BJ, Rizvi SMA, Qu CF, Smith RC. Targeted alpha therapy approach to the management of pancreatic cancer. Cancers: Special Issue "Pancreatic Cancer" 2011;3: 1821-1843.

37. Song EY, Qu CF, Rizvi SM, Raja C, Beretov J, Morgenstern A et.al. Bismuth-213 radioimmunotherapy with C595 anti-MUC1 monoclonal antibody in an ovarian cancer ascites model. Cancer Biol Therapy 2008;7: 76-80.

38. Mukhopadhyay P, ChakrabortyS, Ponnusamy MP†, LakshmananI, Jain M, Batra SK. Mucins in the Pathogenesis of Breast Cancer: Implications in Diagnosis, Prognosis and Therapy. Biochem Biophys Acta 2011; 1815(2): 224-240.

39. Chang F, Qu Yan, J Song, Syed M.A. Rizvi, Yong Li, Ross Smith, Alan Perkins et.al. In vivo and in vitro inhibition of pancreatic cancer growth by targeted alpha therapy using 213Bi-CHX.A"-C595, Cancer Biology \& Therapy 2005; 4 (8): 848-853, doi: 10.4161/cbt.4.8.1892.

40. Price M.R. - Rye P.D, Petrakou E, Murray A, Brady K. et al. Summary Report on the ISOBM TD-4 Workshop: Analysis of 56 Monoclonal Antibodies against the MUC1 Mucin. Tumor Biol 1998;19 (suppl 1):1-20.

41. Qu Chang Fa, Li Y, Song E, Rizvi SMA, Zhang D, Samra J, Smith R, Perkins AC, Apostolidis C, Allen BJ. MUC1 expression in primary and metastatic pancreatic cancer cells for in vitro treatment by 213Bi-C595 radioimmunoconjugate. Brit J Cancer 2004; 91: 2086-2093.

42. Allen B. Systemic Targeted Alpha Radiotherapy for Cancer. Journal of Biomedical Physics \& Engineering 2013;3(3):67-80.

43. S hah M, Silva R Da, Gravekamp C, Libutti S K, AbrahamT and Dadachova E. Targeted radionuclide therapies for pancreatic cancer. Cancer Gene Therapy 2015;22: 375-379 | doi:10.1038/cgt.2015.32.

44. Rizvi SMA, Sarkar S, Groozee G, Allen BJ. Radioimmunoconjugates for targeted alpha therapy for metastatic melanoma. Melanoma Res 2000;10:281-289.

45. Allen BJ, Raja KC, Rizvi S, Yong Li et al Intralesional targeted alpha therapy for metastatic melanoma. Cancer Biol Therapy 2005;4:1318-1324.

46. Santa Cruz GA. Microdosimetry: Principles and applications. Reports of Practical Oncology \& Radiotherapy 2014 Nov 4:www.sciencedirect.com/science/. ../S1507136714001710.

47. Allen, Barry J., Raymond Clarke, and Huang Chenyu. "Targeted Alpha Therapy: From Alpha to Omega." 2013:www.iaea.org/.../45091406.pdf. International Atomic Energy Agency

48. Therasse P, Arbuck SG, Eisenhauer EA, Wanders J, Kaplan RS, Rubinstein L, et al. New guidelines to evaluate the response to treatment in tumors. J Natl Cancer Inst 2000;92:2015-216 (cross ref) solid. 